\title{
IceTop as Veto for IceCube
}

\section{The IceCube Collaboration ${ }^{\dagger}$,}

${ }^{\dagger}$ http://icecube.wisc.edu/collaboration/authors/icrc15_icecube

E-mail: delia.tosi@icecube.wisc.edu

The IceCube neutrino observatory includes a surface array, IceTop, designed to detect and study cosmic rays. This array, located directly above IceCube, can be used to distinguish astrophysical neutrinos from atmospheric neutrinos and penetrating muons, increasing the effective volume of the IceCube detector for the southern sky. In this contribution we present the efficiency of such a veto technique as a function of energy, and compare data and simulation. In particular we focus on one event which was found in a separate analysis (starting event search) in IceCube and passing through IceTop and we study the probability of this event being background.

Corresponding authors: K. Jero ${ }^{1}$, D. Tosi ${ }^{* 1}$,

${ }^{1}$ Dept. of Physics and Wisconsin IceCube Particle Astrophysics Center, University of Wisconsin, Madison, WI 53706, USA

The 34th International Cosmic Ray Conference,

30 July- 6 August, 2015

The Hague, The Netherlands

${ }^{*}$ Speaker. 


\section{Introduction}

The IceCube observatory, completed in 2010, is a particle detector located at the geographic South Pole [1]. The experiment features two major detectors, one buried in ice and the other on the ice surface. The in-ice neutrino telescope, IceCube, consists of 86 strings equipped with 60 optical sensors each, instrumenting a volume of a cubic kilometer. The optical sensors are installed between depths of $1450 \mathrm{~m}$ and $2450 \mathrm{~m}$ in the ice sheet. The IceTop air shower array consists of 81 stations located near the top of each in-ice string. A station consists of two frozen water tanks, each equipped with two optical sensors. The optical sensors (digital optical modules, DOMs) of both arrays detect the Cherenkov radiation emitted by secondary charged particles. On the surface, these are charged leptons produced in hadronic or electromagnetic interactions in the atmosphere. In ice, these are either penetrating muons from showers, or particles produced by neutrino interactions in the Antarctic ice cap or in the bedrock.

The discovery of an astrophysical neutrino flux [2] was achieved in 2013 by using the outer layer of the in-ice detector as a veto. This technique allows high energy events to be distinguished as starting inside the detector or coming from the outside, with the former indicating the events are astrophysical neutrino interactions. Variations of this method have been implemented to improve sensitivity and widen its energy range [3]. IceTop was designed to measure the spectrum and composition of cosmic ray particles in the energy range between $10^{14}$ and $10^{18} \mathrm{eV}$, exploiting the relationship between air shower energy and the energy of penetrating muons (as detected by the in-ice detector) [4]. In this paper we investigate the possibility of using IceTop as a veto for IceCube. The location of the detector makes it a suitable instrument to tag air shower particles which accompany muons that penetrate deep into the ice. This veto could create a background free phase space for down-going astrophysical neutrinos undergoing a charged current interaction between IceTop and the in-ice detector, which are currently not detectable by any other analysis. This study aims to determine the efficiency of IceTop as a veto for IceCube, and its energy threshold, with an improved methodology as compared to [5]. In particular this analysis focuses on events which cross both the IceCube array and IceTop. We call these events "vertical events".

While this analysis was being developed, a vertically down-going muon neutrino was found in the starting event analysis [6]. The interaction vertex is reconstructed near the horizontal center of the detector, after the neutrino candidate has passed more than 10 layers of DOMs. The outgoing muon travels nearly vertically and deposits over $400 \mathrm{TeV}$ in the detector. It is highly improbable that the particle whose interaction generated the event was a muon which did not emit detectable amounts of light before the reconstructed vertex. Additionally, the findings of [7] show that only neutrinos produced by charmed mesons in the atmosphere can be a background to this event. From dedicated simulations with CORSIKA [8] utilizing the DPMJET II.5 [9] hadronic model, a background of one event per $\sim 390.5$ years was found to be accompanied by a muon bundle with less than $300 \mathrm{GeV}$ energy. Since DPMJET II.5 is known to overproduce charm muons and neutrinos, this simulation alone provides a conservative estimate that this event is a $3 \sigma$ deviation from background. As such, it is interesting to consider this event within the framework of an IceTop veto. In the following sections data selection, background and signal estimation are presented. The presented analysis was performed on 102 days of data between 2012 and 2014. 


\section{Data selection}

The data collected by the IceCube observatory is transferred to the northern hemisphere by satellite and therefore must be greatly reduced in rate before transmission. Several filters are in place to select events which are interesting for one or the other analysis. In the analysis presented here the events of interest are down-going bright vertical events which pass through both IceTop and IceCube. The most suitable stream for this analysis is the one containing events which triggered the in-ice detector with a charge greater than one thousand photoelectrons (PE). The typical trigger rate for this stream is between 1 and $2 \mathrm{~Hz}$. Events passing very close to a DOM may deposit a disproportionate amount of charge into that DOM with respect to the charge detected in all the other DOMs, leading to an out-of-ordinary value of the charge for events which are otherwise not very bright. To account correctly for this effect, the total number of photoelectrons is recalculated by removing the DOMs which have more than $50 \%$ of total charge. This quantity is called "homogenized total charge" (Qtoth). Passing events are required to have Qtoth $>1000$ PE.

The direction of each event is reconstructed using a likelihood-based algorithm (see section 3). The track is required to have a length greater than $800 \mathrm{~m}$ in IceCube (as

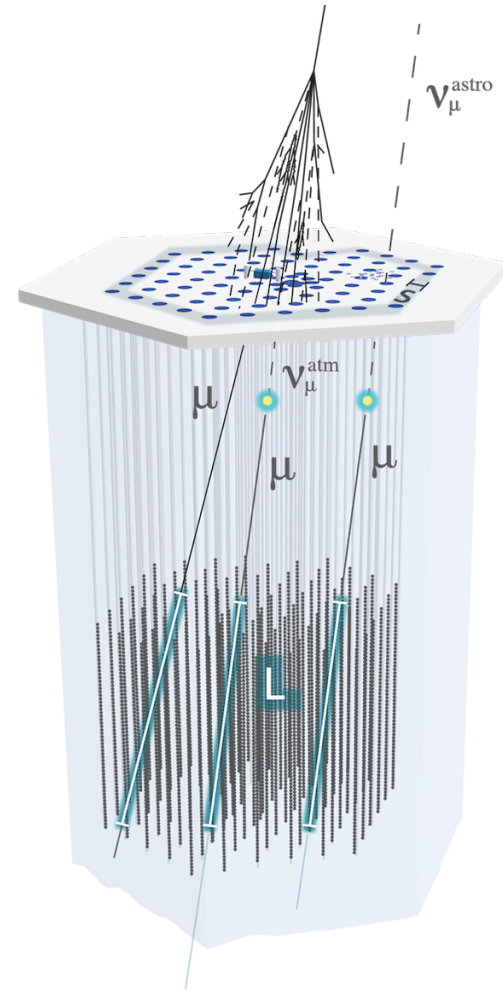

Figure 1: Illustration of the cuts used to select events for this analysis: $\mathrm{L} \geq 800 \mathrm{~m}$ and $\mathrm{S} \geq 75 \mathrm{~m}$. measured along the reconstructed direction) and a trajectory such that the extrapolated intersection with the ice cap surface (impact point) lies inside the perimeter of IceTop by at least $75 \mathrm{~m}$. This series of cuts reduces the data rate to $2.8 \%$ of the original trigger level where only $1000 \mathrm{PE}$ in ice was required. Events selected by these containment cuts are muon tracks reconstructed to sub-degree angular resolution. A sample of extrapolated impact points and the distribution of reconstructed zenith angles for all events are shown in fig. 2 and fig. 3 respectively.

The time of impact at each DOM on the surface $\left(t_{0 i}\right)$ is calculated for a hypothetical shower generating the reconstructed track of the muon/muon bundle. Pulses recorded by IceTop DOMs are then processed to remove redundant information, leaving a unique time for each hit tank. The difference $\left(t_{i}-t_{0 i}\right)$ between the hit time $\left(t_{i}\right)$ and the expected arrival time of the shower $\left(t_{0 i}\right)$ is calculated, and shown as the distribution in fig. 4.

Most of the hits are recorded at the predicted time and in a window of a few hundred nanosecond afterwards. This distribution is used as a template to establish the likelihood of a hit to be correlated with a shower, rescaled so that the maximum value is one. In this construction, a hit in perfect time will be counted as one hit while early and late hits will be penalized proportionally to how often they are observed to occur. This smooth weighting strategy does not require an arbitrary time window but naturally takes into account all the hits recorded (an improvement compared to [5]). The sum of weighted IceTop hits ("wHits") produces a floating value for each event which 
can span from 0.0 (no tanks having any hit) to 162.0 (all tanks having a hit at the shower time).

To determine a proxy to the muon energy, the expected number of photons is fitted via an analytic template which scales with the energy of the muon. This calculation leads to an energy estimator, called "MuEx" [10], which is more sophisticated than Qtoth and accounts for energy losses outside the detector. In the following, this energy proxy is given in units which can be roughly interpreted as $\mathrm{GeV}$, but this should be not interpreted as the true value of the energy of the muon or muon bundle. A calibration to muon energy needs a high statistics simulation. In another analysis the value of MuEx has been found to correspond to about half the energy of the muon/muon bundle [11]. However, since the calibration depends on the specific cuts of each analysis, this should not be taken as the correct conversion factor for this analysis.
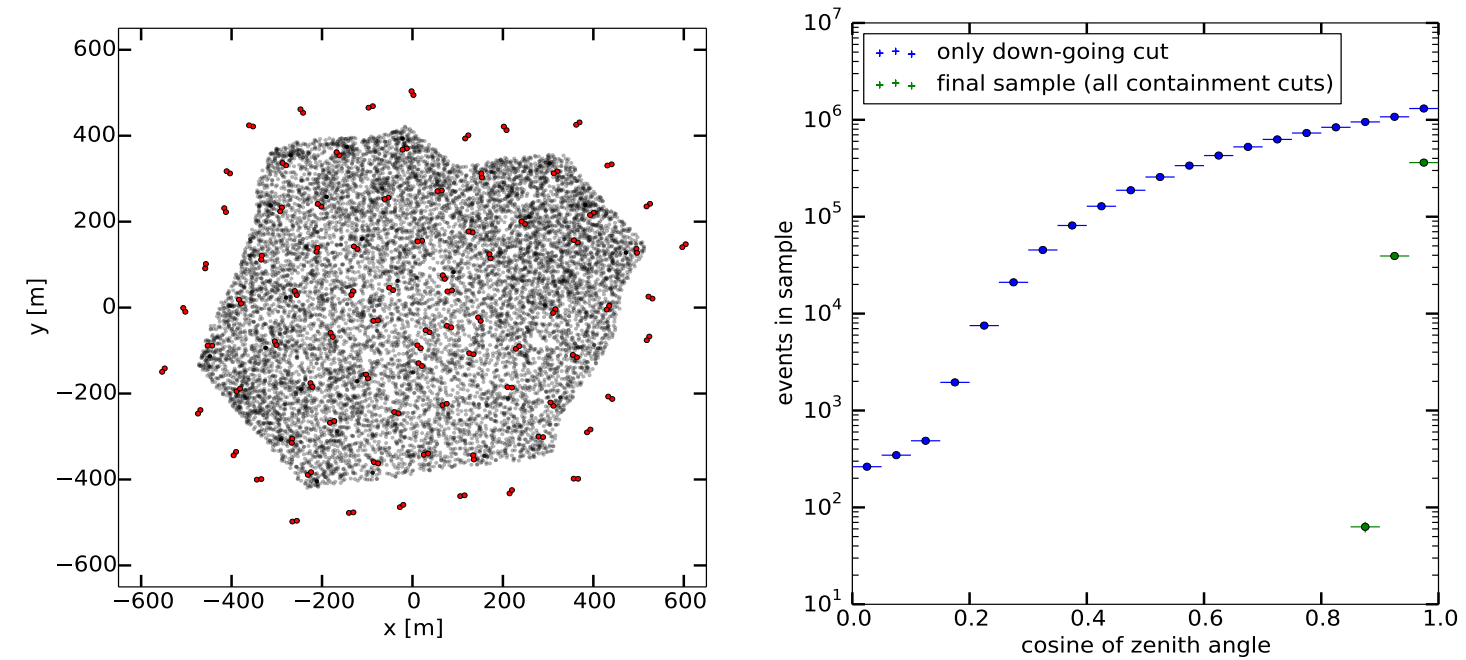

Figure 2: Distribution of a sample of selected Figure 3: Distribution of events as a function events in surface coordinates. of cosine of zenith angle for down-going events and for the final sample which selects vertical events $(\cos (\theta)>0.85)$.

\section{Simulation}

In order to save time and space in generating a simulation set, the generation was targeted to mimic the background of the vertically down-going starting track discussed in section 1 . The air shower simulation code CORSIKA [8] was modified and used to generate the particles at ground which were then simulated as events in the detectors using the standard IceTop and IceCube simulation tools. The development of the shower was modified to simulate the hadronic and muonic portions while holding the electro-magnetic component. If the total muon energy was found to be below the deposited energy of the specimen event, then the shower was discarded and the propagation time saved. DPMJET II.5 was used for the reasons mentioned in section 1.

The dataset produced, equivalent to 0.1 year in the phase space relevant to the candidate neutrino (see section 4), was used to validate this new simulation technique and for reconstruction verification. Although this test set is not sufficient to serve the scope of the analysis presented here, it was used to compare the performance of different reconstruction algorithms. The best performance in terms of time extrapolation and zenith reconstruction was found to be achieved by a 
two-step reconstruction algorithm. A likelihood-based algorithm, performed in the offline processing of the stream selected at trigger level, is used as best guess for direction and vertex position. This algorithm fits the first arrival times and charge of detected photoelectrons on all the DOMs in the detector, assuming that the arriving light is originating from a muon moving at the speed of light and emitting Cherenkov radiation. The Cherenkov radiation looses intensity as it moves away from the muon, according to a simple analytic parameterization of photon propagation in ice. This reconstructed direction is then used as seed for a second fitting algorithm, which fits for the time of the interaction using only the time of the first unscattered photon and the total charge in each DOM. The combination of these steps provides better agreement between true and reconstructed values in simulation.

\section{Background estimation}

The distribution of events in the space of wHits and energy proxy MuEx is shown in fig. 5, normalized (for visualization purpose) by the events in each MuEx bin. At low energy, there appears to be a turn-on feature which is caused by selecting data on a different energy proxy (Qtoth) (see section 2). The value of wHits is correlated with the energy since higher energy showers will produce on average more hits in IceTop synchronously with the shower. The spread of wHits is also reduced at high energy as the total value of wHits is dominated by the high-weight hits.
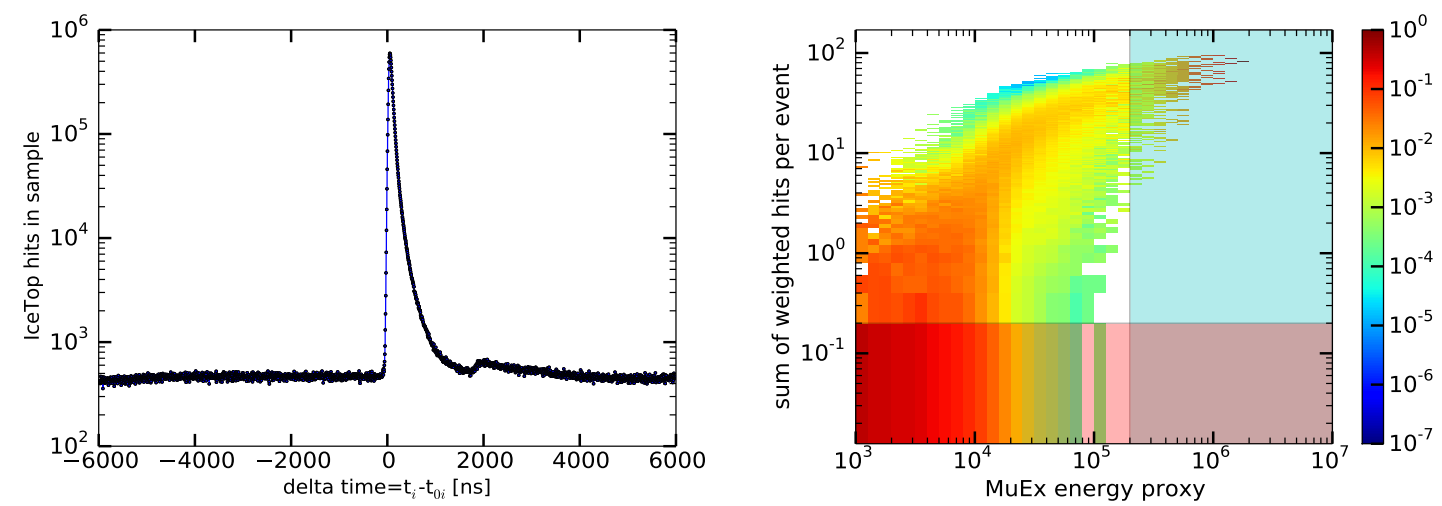

Figure 4: IceTop hit times minus arrival time of Figure 5: 2D histogram of all the events selected shower at surface, as extrapolated from the fit as a function of MuEx and IceTop weighted hits. performed on pulses recorded in ice. The weight For visualization reasons, the histogram is norof an IceTop hit is calculated from this template malized by the number of entries in each energy after rescaling it so that the maximum is 1 .

bin. For shaded areas refer to text.

A detected flux from astrophysical neutrinos would populate a specific area in the phase space of high energy and low wHits (bottom right of the histogram) since astrophysical neutrinos would have IceTop hits only due to random coincidences and noise hits. In our sample, no cosmic ray events above $\mathrm{MuEx} \approx 2 \times 10^{5}$ have less than $\mathrm{wHits}=1$.

The distribution of showers observed in the sample here analyzed appears to be steeply falling towards the region of interest. Rare events (such as muons from prompt decay, or unusual showers which develop after an early or a late interaction, creating exceptional muons), not visible in the 
sample, may disrupt the distribution or introduce a change in slope. In order to properly estimate the background of cosmic rays in the region of interest, a comprehensive simulation including all these phenomena with high statistics is needed. Since no such simulation has been produced so far, it is conceivable to attempt to extrapolate the background into the region of interest from the data, assuming that the population of cosmic rays follows smoothly the distribution observed.

To do so, two methods can be envisioned. The first method is based on looking at the wHits distribution of showers with an energy proxy value above a certain threshold: from this distribution, mostly concentrated at high wHits, one can extrapolate the rate of events expected at low wHits. This means integrating in energy to the right of the vertical line in the fig. 5 (green shaded area) and projecting to the wHits axis. The second method is based on looking at the energy distribution of showers with less than a maximum acceptable value of wHits (for example 0.2): from this distribution one can extrapolate the expected rate for a certain value of energy proxy. This is equivalent to integrating the 2-dimensional distribution in wHits from 0.0 up to the chosen value of wHits (red shaded area in fig. 5) and then projecting it on the energy proxy axis.

The two methods should provide a consistent estimation of the background for the same bin. The first method, however, is difficult to apply because of the low statistics at high energy and because of the large gap between the data and extrapolation region. In addition, the lack of a proper fit model makes any fit of the data difficult. The second method offers the advantage of higher statistics and is therefore more robust and reliable with the current set of data analyzed.

\section{Signal estimation}

Neutrino-generator, a Monte Carlo simulation program based on ANIS [13] with CTEQ cross section tables is used to generate neutrinos and propagate them through the Earth. The neutrinos are forced to interact before passing through the detector and are assigned a weight. For this analysis a general purpose dataset of muon neutrinos with a spectrum of $\mathrm{E}^{-1}$ over an angular range of $0^{\circ} \leq \theta \leq 180^{\circ}$ and energy range of $10^{2} \mathrm{GeV} \leq E_{v} \leq 10^{7} \mathrm{GeV}$ was used. The same data selection criteria as explained in section 2 was applied. To obtain the signal spectrum (shown in fig. 6) the

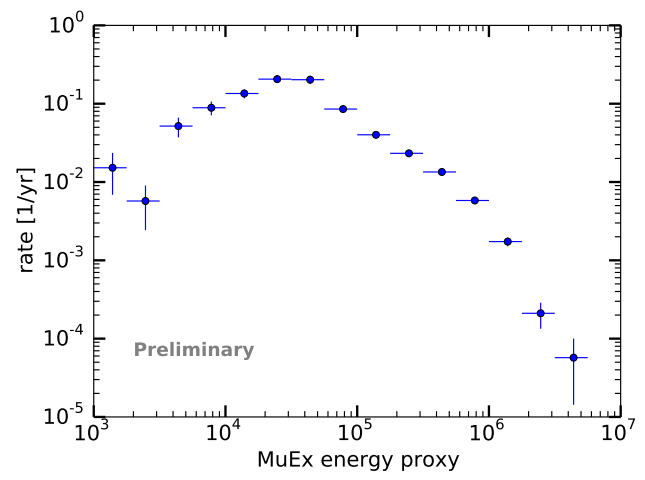

Figure 6: Expected neutrino rate (from simulation) vs reconstructed energy proxy using the best fit astrophysical flux from [12]. surviving events were re-weighted to the best fit astrophysical neutrino spectrum of [12] $E^{2} \phi(E)=1.5 \times 10^{-8}(\mathrm{E} / 100 \mathrm{TeV})^{-0.3} \mathrm{GeV} \mathrm{cm}^{-2} \mathrm{~s}^{-1} \mathrm{sr}^{-1}$. From the observed event rate the expected number of events above a certain energy threshold was calculated.

\section{Sensitivity}

By lowering the maximum allowed value of wHits, it is possible to increase the rejection power of the veto and lower the energy threshold above which the veto is efficient. The trend is shown in 

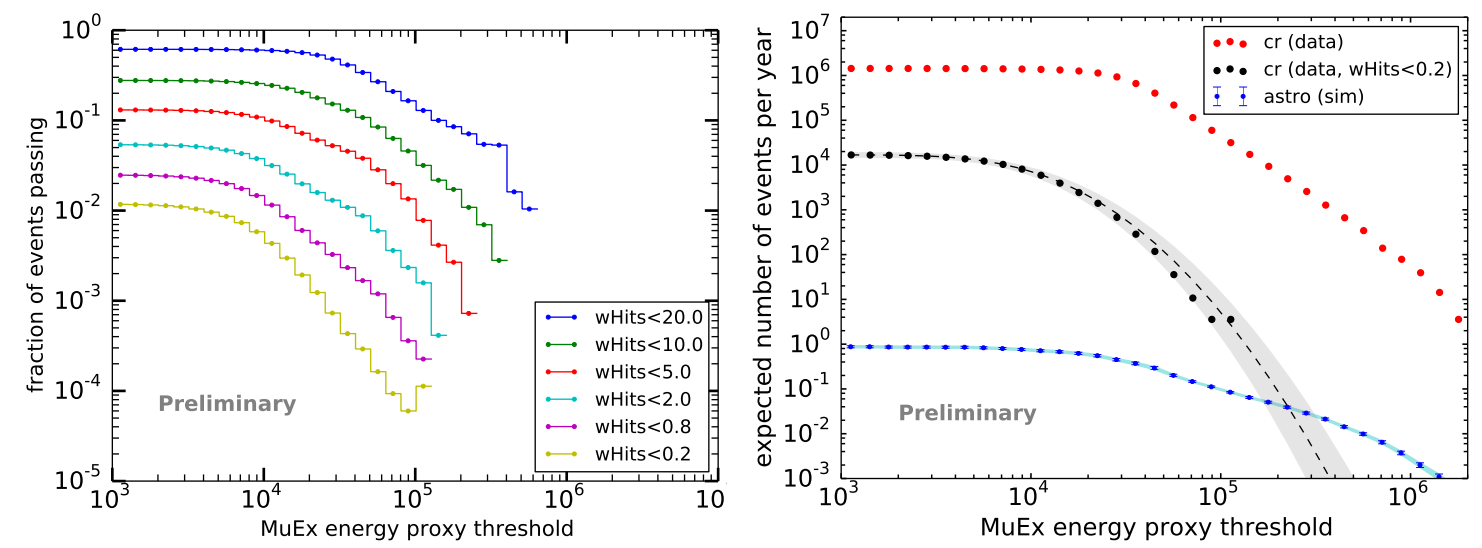

Figure 7: Fraction of events with less than a Figure 8: Observed background rate and excertain value of wHits. pected neutrino signal versus energy proxy threshold.

fig. 7 for some example values of wHits. Fig. 8 shows the number of expected neutrino (blue line) and background events (black dots) above a certain threshold for the energy proxy, rescaled to one year, for events with a wHits value less than 0.2. The total number of events in the selection (i.e. all the events shown in fig. 5) is shown in red. The energy threshold at which the signal expectation exceeds the background is roughly $2 \times 10^{5} \mathrm{MuEx}$, and the expected number of neutrino events is 0.1 per year, primarily due to the very small solid angle to which this analysis is sensitive.

No events in the sample exceed the $5 \sigma$ error band of the background estimation. The brightest event in the sample with wHits $<0.2$ has a MuEx value of $104 \times 10^{3}$ and a wHits value of 0.03 . The bright vertical event found by the starting event analysis has a MuEx value of $180 \times 10^{3}$ and 13 hits for a value of wHits of less than 0.01 , so it would be slightly above the background.

For comparison fig. 9 shows the event viewer of the muon neutrino candidate, while fig. 10 shows a shower of similar energy which lights up IceTop (wHits $\approx 47$ ) and is reconstructed by IceTop as having an energy of $7.5 \mathrm{PeV}$.

\section{Outlook}

Several improvements to this analysis are under investigation. The value of wHits can be optimized with a more detailed study of the background. The distance of a hit DOM from the impact point can be used as additional information in establishing the weight of each hit. The effect of the angular resolution of individual events on the veto probability is under study. Higher statistics might make possible the extrapolation of background along the wHits axis and lead to increased analysis efficiency.

This analysis allows for the first time a measurement of the veto potential of IceTop against cosmic ray muon and neutrino background. A proper understanding using data and simulation will allow for reliable predictions of the veto efficiency of a large $\left(\sim 100 \mathrm{~km}^{2}\right)$ array as is currently envisioned for a future upgrade of IceCube [14] [15] [16]. 

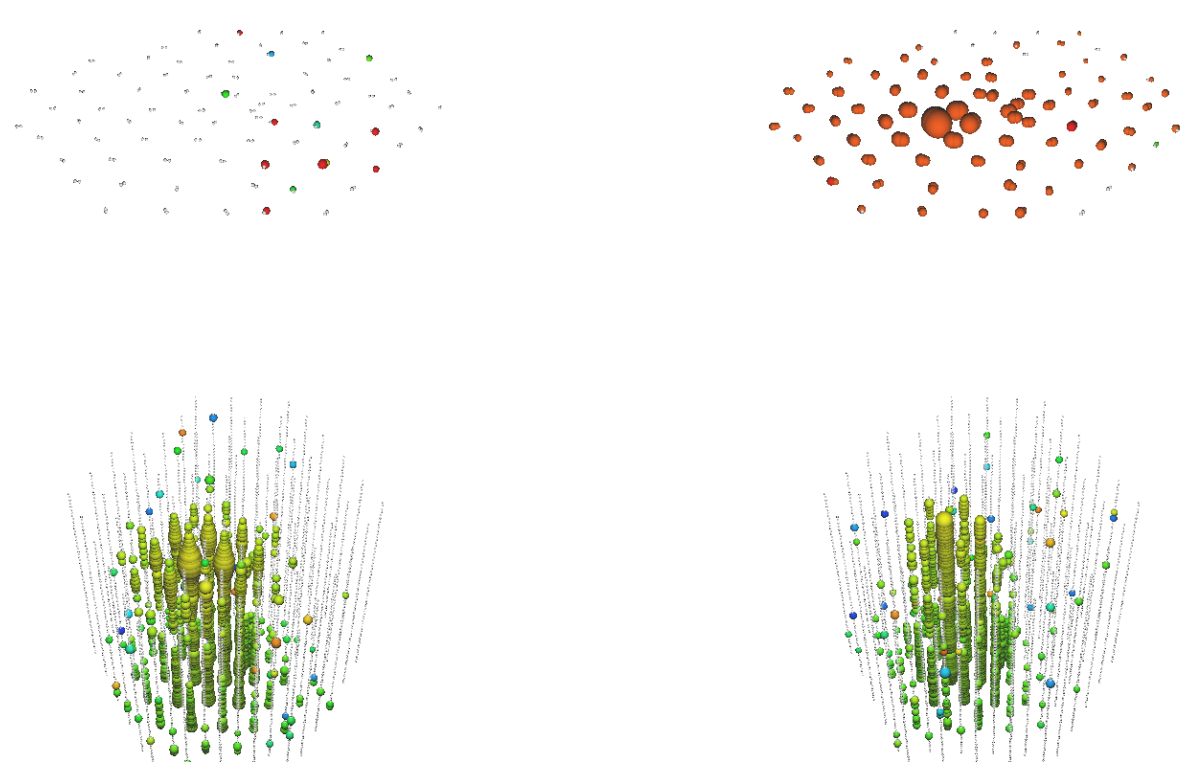

Figure 9: Event viewer of the candidate muon Figure 10: Event viewer of a typical shower neutrino event starting in the detector, found which produces a muon bundle of energy simwith the starting track analysis. The dot colors ilar to the energy of the neutrino candidate event indicate the relative time of hits (red: first, blue: in fig. 9. MuEx value is $\approx 180 \times 10^{3}$ and wHits last), the size indicates the amount of charge de- is 47 .

tected by each DOM. MuEx value for this event is $\approx 180 \times 10^{3}$, wHits is 0.03 .

\section{References}

[1] IceCube Collaboration, A. Achterberg et al., Astropart. Phys. 26 (2006) 155.

[2] IceCube Collaboration, M. G. Aartsen et al., Science 342 (2013) 1242856.

[3] IceCube Collaboration, M. G. Aartsen et al., Phys. Rev. D 91 (2015) 022001.

[4] IceCube Collaboration, PoS(ICRC2015)334, these proceedings.

[5] IceCube Collaboration, M. G. Aartsen et al., The IceCube Neutrino Observatory Part VI: Ice Properties, Reconstruction and Future Developments, Proc. 33rd ICRC.

[6] IceCube Collaboration, PoS(ICRC2015)1081, these proceedings.

[7] A. K. T. Gaisser, K. Jero and J. van Santen, Phys. Rev. D 90 (2014) 23009.

[8] J. C. G. S. D. Heck, J. Knapp and T. Thouw, Tech. Rep. FZKA 6019 (1998).

[9] J. Ranft, Phys. Rev. D 51 (1995) 64.

[10] IceCube Collaboration, M. G. Aartsen et al., J. Inst. 9 (2014) P03009.

[11] IceCube Collaboration, M. G. Aartsen et al., arXiv:1507.0400.

[12] IceCube Collaboration, M. G. Aartsen et al., Phys. Rev. Lett. 113 (2014) 101101.

[13] A. Gazizov and M. P. Kowalski, Comput. Phys. Commun. 172 (2005) 203.

[14] IceCube Collaboration, PoS(ICRC2015)1146, these proceedings.

[15] IceCube Collaboration, PoS(ICRC2015)1070, these proceedings.

[16] IceCube Collaboration, PoS(ICRC2015)1156, these proceedings. 